\title{
The Development of Self-Awareness and Relationship to Emotional Functioning During Early Community Reintegration After Traumatic Brain Injury
}

\author{
Jennifer M. Fleming \\ The University of Queensland and Occupational Therapy Department, Princess Alexandra Hospital
}

Heidi T. Winnington, Azaria J. McGillivray, Boyana Ana Tatarevic

and Tamara L. Ownsworth

Occupational Therapy Department, Princess Alexandra Hospital

\begin{abstract}
Impaired self-awareness may affect clients' emotional status, engagement in Irehabilitation and community reintegration following traumatic brain injury (TBI). The study aimed to investigate the relationship between self-awareness, emotional distress and community integration in adults with TBI during the transition from hospital to the community. Thirty-four rehabilitation clients with TBI were assessed in the week before and 2 months after discharge home. Measures of self-awareness and emotional functioning were administered predischarge and repeated at follow-up along with a measure of community integration. Nonparametric tests were used to compare levels of self-awareness and emotional distress pre- and postdischarge, their interrelationships and association with community integration. Self-awareness significantly increased following discharge, and a trend towards increased depression was found. There were no consistent relationships found between level of self-awareness, emotional functioning, and community integration. The development of self-awareness in the immediate postdischarge phase suggests this is an important time for clinical interventions targeting compensation strategies and adjustment to disability.
\end{abstract}

\begin{abstract}
Disorders of self-awareness are common following traumatic brain injury (TBI), and have consequences for clients' engagement in rehabilitation, emotional status, and integration into the community (Andersson, Gundersen, \& Finset, 1999; Fischer, Gaugel, \& Trexler, 2004; Fordyce \& Roueche, 1986; Sherer Oden, Bergloff, Levin, \& High, 1998b; Wallace \& Bogner, 2000). According to Prigatano and Schacter (1991), self-awareness is 'a process requiring integration of information from the external reality and inner experience' (p. 10). Although the proportion of individuals with disorders of self-awareness is unknown, Sherer,
\end{abstract}

Bergloff, Levin, High, Oden and Nick (1998a) noted that most individuals with a TBI who are receiving postacute rehabilitation services display impaired self-awareness to some degree. The consequences of poor self-awareness after TBI can include low motivation, noncompliance, minimal engagement, and lack of progress in therapy (Fischer et al., 2004; Fleming, Strong, \& Ashton, 1996; Lanham, Weissenburger, Schwab, \& Rosner, 2000; Malia, 1997).

The transition from hospital to home is a notably emotional and challenging phase for people with TBI that involves participation in 
activities of daily living away from the structure and therapeutic support of the rehabilitation setting for the first time. Researchers generally suggest that it is during the transition process that an individual's awareness of deficits typically increases and is associated with emotional distress (Fordyce \& Roueche, 1986; Godfrey, Partridge, Knight, \& Bishara, 1993; Malec \& Moessner, 2000). Emotional reactions such as depression and anxiety may lead to reduced motivation and participation in therapy and impede further community integration (Fleming, Strong, \& Ashton, 1998; Langer \& Padrone, 1992). However, research is yet to systematically examine the development of self-awareness and emotional changes during the critical transition period from the hospital to home environment. Therefore, this longitudinal study of individuals with TBI aims to examine levels of self-awareness and emotional functioning prior to discharge and 2 months following discharge and their association with community reintegration.

\section{Self-Awareness and Recovery from Traumatic Brain Injury}

Self-awareness is a major consideration for health professionals due to its association with motivation and functional outcomes for individuals in brain injury rehabilitation. As reported by Prigatano (1992), 'patients cannot maintain a productive lifestyle unless they have come to face the realities of their life and this means improving self-awareness and self-acceptance' (p. 60). In a study of selfawareness, goal setting and rehabilitation outcome, Fischer et al. (2004) identified that individuals with TBI $(n=68)$ who had low self-awareness typically had unrealistic goals and expectations and, consequently, experienced poorer rehabilitation outcomes. Others have also noted that impaired self-awareness reduces the ability to adopt compensatory strategies to counteract impairments that impact upon functional outcomes following TBI (Dirette, 2002; Fleming \& Strong, 1999).

A consistent pattern of increasing self-awareness has been described as time progresses following TBI (Fischer et al., 2004; Langer \& Padrone, 1992). For example, in a longitudinal study of 55 participants with TBI, Fleming and Strong (1999) found that level of self-awareness improved for most areas of function over the first year after injury. In a cross-sectional study of 66 participants with TBI who were between 6 months and 3 years postinjury, Godfrey et al. (1993) found that level of self-awareness increased according to time since injury. The development of more accurate selfappraisal during TBI recovery was attributed to life experiences and opportunities to identify problems in daily activities. However, the development of self-awareness can differ between individuals over varying time periods (Dirette, 2002; Fleming \& Strong, 1997). Participants in a qualitative study (Dirette, 2002) reported that the development of self-awareness occurred through a comparison of premorbid function to current function, which was triggered by events or incidents. Some participants in Dirette's study reported that while repeated verbal information was given to them by rehabilitation staff to increase their awareness of deficits, it was through actual participation in functional tasks in a familiar environment following hospital discharge that deficits were first personally identified. Overall, previous research generally suggests that individuals' level of self-awareness increases over time as a result of participating in familiar activities in the community.

\section{Self-Awareness and Emotional Functioning}

Although increased self-awareness has been associated with improved motivation, participation in rehabilitation and the adoption of compensatory strategies, it also appears to be related to an increase in emotional distress (Andersson et al., 1999; Dirette, 2002; Fleming et al., 1998; Fordyce \& Roueche, 1986; Malec \& Moessner, 2000; McGlynn \& Schacter, 1989; Sherer et al., 1998a; Wallace \& Bogner, 2000). Emotional reactions related to increased self-awareness may include depression, anxiety, or even psychosis (Giacino \& Cicerone, 1998). Fischer et al. (2004) noted that along with depressive symptoms, a decrease in self-efficacy is common in individuals with heightened self-awareness. Secondary frustration may also occur, as individuals become more aware of their deficits (Fleming \& Strong, 1995).

In a study comparing individuals' emotional status following TBI, levels of emotional distress were the highest for the first year and 2 to 3 years postinjury groups compared to a 6 months postinjury group who displayed greater deficits in awareness (Godfrey et al., 1993). Fleming and Strong (1997) presented three case studies that illustrated a relationship between high self-awareness, greater motivation and increased emotional distress in some clients. Katz, Fleming, Hart-Maeir, Keren and Lightbody (2002) also demonstrated the relationship between these variables in a series of case studies. Using cluster analysis with 55 individuals with TBI, Fleming et al. (1998) provided support for the relationship between self-awareness, motivation and emotional distress, although 1-year 
postinjury rehabilitation outcomes did not differ significantly between the high and low self-awareness groups. In contrast, Malec and Moessner (2000) found that impaired self-awareness did not correlate with increased emotional distress following implementation of a comprehensive day treatment program which incorporated self-awareness training, suggesting that interventions may effectively support individuals' emotional adjustment as awareness of deficits develops.

As people with TBI move beyond the hospital environment, they may be at greater risk of emotional distress as they begin to identify functional deficits in the home and work environment and understand the implications of their injury (Wallace \& Bogner, 2000). In a study investigating self-awareness and emotional adjustment during the transition from the hospital to the community, Fleming, Connell, Tooth and Strong (2002) found significantly greater impairment of self-awareness in individuals with TBI $(n=23)$ compared to individuals with spinal cord injuries (SCI) $(n=13)$. Self-awareness was observed to increase in both groups within the 2-month period from discharge, thus supporting the suggestion that self-awareness increases with participation in real life experiences. Level of emotional distress also increased for both groups, although this was only significant for the SCI group. However, this study did not investigate the association between self-awareness and emotional functioning. Therefore, the present study aimed to empirically investigate self-awareness in individuals with TBI and its relationship with emotional functioning during the early community integration phase.

\section{Community Integration}

The term 'community integration' encompasses some combination of participation in the three domains of social, home and vocational roles (Tate, Hodgkinson, Veerabangsa, \& Maggiotto, 1999; Willer, Rosenthal, Kreutzer, Gordon, \& Rempel, 1993). While self-awareness is considered a positive factor for engaging in and achieving rehabilitation outcomes, it has been proposed that the heightened emotional distress associated with improved self-awareness may impede levels of community integration (Ben-Yishay, Silver, Piasetsky, \& Rattock, 1987; Brown, Gordon, \& Spielman, 2003; Lanham et al., 2000; LoBello, Underhil, Valentine, \& Stroud, 2003).

In contrast, previous research (Malec \& Moessner, 2000; Ownsworth \& McFarland, 2004; Sherer et al., 1998a) has identified that an increase in levels of self-awareness following TBI may produce better community reintegration. Sherer et al. (1998a) reported that accurate self-awareness resulted in more favourable vocational outcomes postdischarge. An empirical study by Ownsworth and McFarland (2004) found that an increase in the level of self-awareness in the context of rehabilitation was associated with improved social interaction and emotional wellbeing. In other studies increased levels of self-awareness have also been associated with successful independent living outcomes (Malec \& Moessner, 2000; Trudel, Tryon, \& Purdum, 1998) and employability (Ben-Yishay et al., 1987; Sherer, Hart, Nick, Whyte, Thompson, \& Yablon, 2003). It has been suggested that the implementation of rehabilitation programs postdischarge may improve levels of self-awareness and emotional functioning, positively impacting on functional outcomes (BenYishay et al., 1987; Malec \& Moessner, 2000).

Overall, there is limited research on the relationship between self-awareness, emotional functioning and community integration following the transition from hospital to home. Predominantly, previous research has conducted cross-sectional analyses to assess the links between self-awareness and emotional functioning. Although previous studies suggest that more accurate self-appraisal contributes to better community integration, this relationship needs to be understood in the light of the potential contribution made by emotional changes associated with the development of self-awareness.

\section{Aims}

The main aims of this study were (a) to examine levels of self-awareness, emotional functioning, and community integration in adults with TBI during the transition from inpatient rehabilitation to the home and community environment; (b) to examine change in self-awareness and emotional functioning over this period; and (c) to examine the associations between these variables. Based on previous research, it was hypothesised that level of self-awareness would increase over the period of transition from hospital to the community. The second hypothesis was that greater self-awareness would be associated with higher levels of emotional distress. A third hypothesis was that greater self-awareness would be associated with higher levels of integration into the community.

\section{Method}

\section{Participants}

Participants consecutively discharged from an inpatient brain injury rehabilitation unit of a major 
Table 1

\begin{tabular}{|c|c|}
\hline \multicolumn{2}{|c|}{$\begin{array}{l}\text { Diagnostic and Demographic Characteristics } \\
\text { of the Participants With TBI }(n=34)\end{array}$} \\
\hline Characteristic & $N(\%)$ \\
\hline \multicolumn{2}{|l|}{ Gender } \\
\hline Male & $29(85.3 \%)$ \\
\hline Female & $5(14.7 \%)$ \\
\hline Age (years) & $\begin{array}{c}A=26.4, S D=10.9, \\
\text { range } 17-58\end{array}$ \\
\hline \multicolumn{2}{|l|}{ Cause } \\
\hline Motor accident & $17(50 \%)$ \\
\hline Fall from fixture & $5(14.7 \%)$ \\
\hline Fall from horse & $4(11.8 \%)$ \\
\hline Pedestrian & $3(8.8 \%)$ \\
\hline Assault & $2(5.9 \%)$ \\
\hline Sporting accident & $2(5.8 \%)$ \\
\hline Other trauma & $1(2.9 \%)$ \\
\hline \multicolumn{2}{|l|}{ Premorbid occupation } \\
\hline Tradesperson & $9(26.5 \%)$ \\
\hline Labourers and related & $7(20.6 \%)$ \\
\hline Plant machine operator and driver & $4(11.8 \%)$ \\
\hline $\begin{array}{l}\text { Salesperson and personal } \\
\text { service worker }\end{array}$ & $4(11.8 \%)$ \\
\hline Student & $3(8.8 \%)$ \\
\hline Professional & $3(8.8 \%)$ \\
\hline Unemployed & $2(5.9 \%)$ \\
\hline Homemaker & $2(5.9 \%)$ \\
\hline Education (in years) & $\begin{array}{l}M=11.8, S D=2.6 \\
\text { range } 8-19\end{array}$ \\
\hline \multicolumn{2}{|l|}{ Marital status } \\
\hline Single & $23(67.6 \%)$ \\
\hline Married/defacto & $11(32.4 \%)$ \\
\hline Neurosurgical intervention & $7(20.6 \%)$ \\
\hline Initial Glasgow Coma Score & $\begin{array}{l}M=6.03, S D=3.5 \\
\quad \text { range } 3-14\end{array}$ \\
\hline $\begin{array}{l}\text { Length of posttraumatic } \\
\text { amnesia (days) }\end{array}$ & $\begin{aligned} 1= & 37.8, S D=24.3 \\
& \text { range } 2-105\end{aligned}$ \\
\hline $\begin{array}{l}\text { Length of acute care hospital } \\
\text { stay (days) }\end{array}$ & $\begin{array}{c}1=25.4, S D=15.8, \\
\text { range } 0-67\end{array}$ \\
\hline $\begin{array}{l}\text { Length of inpatient } \\
\text { rehabilitation (days) }\end{array}$ & $\begin{aligned} 1= & 37.8, S D=24.8 \\
& \text { range } 5-105\end{aligned}$ \\
\hline
\end{tabular}

metropolitan public hospital during a period of 7 months in total were invited to participate in the study. Participants were included in the study if they had a medical diagnosis of TBI (based on loss of consciousness, CT scan, Glasgow Coma Score [GCS] or posttraumatic amnesia [PTA]), gave informed consent, and were between 17 and 65 years of age. Participants who were unable to communicate in English (e.g., due to dysphasia and/or non-English speaking), participants with severe cognitive impairment limiting ability to answer questions, as well as participants with a history of previous neurological or major psychiatric disorder were excluded from the study. Participants who would be unavailable for followup 2 months postdischarge were also excluded.

Thirty-four participants with TBI met the inclusion criteria and were recruited into the study. Diagnostic and demographic characteristics of the participants are displayed in Table 1 . The participants were predominantly males $(85 \%)$ with a mean age of 26 years at the time of interview. Of those participants $(n=32)$ with a documented initial GCS, $25(78 \%)$ had severe TBI (GCS 3-8), 4 (12.5\%) had moderate TBI (GCS 9-12), and 3 (9.4\%) had mild TBI (GCS 13-14). However, according to length of PTA, all participants $(n=34)$ had severe TBI, as indicated by a period of PTA greater than 24 hours. At postdischarge two participants could not be contacted for follow-up assessment and a further two did not complete the self-report questionnaires, and therefore their data on these measures were included in pre-discharge analyses $(n=34)$ but not postdischarge analyses $(n=30)$.

\section{Measures}

Self-Awareness of Deficits Interview (SADI). The SADI (Fleming et al., 1996) is an interviewer-scored semi-structured interview that assesses the level of self-awareness in adults with TBI. Self-awareness is measured on three subscales: self-awareness of deficits, selfawareness of the functional implications of deficits, and ability to set realistic goals. The interviewer assigns a score of 0 to 3 for each subscale $(0=$ intact self-awareness and $3=$ severe impairment of self-awareness). A total SADI score is obtained by summing the three subscale scores (range 0-9). The reports of a significant other or treating therapist can be used to assist the interviewer to rate the participant's level of self-awareness. The SADI has high test-retest reliability (ICC $=0.94$ for total score and ICC $=0.85,0.86$ and 0.86 for subscale scores) (Simmond \& Fleming, 2003b), high interrater reliability (ICC $=0.85$ ) (Fleming et al., 1996) and takes approximately 20 to 30 minutes to administer. Extrapolating from the subscale scoring guidelines (see Fleming et al., 1996), a total SADI score of 0 was classified as 'intact self-awareness', and scores of 1 to 3, 4 to 6 , and 7 to 9 as 'mild', 'moderate' and 'severe' impairment of self-awareness respectively, for 
the purposes of describing level of self-awareness for the current sample.

Center for Epidemiology Studies Depression Scale (CES-D). The CES-D (Devins \& Orme, 1985) was developed to evaluate symptoms of depression in the general population by assessing the frequency and duration of depressive symptomatology in the week preceding its administration. The CES-D provides a screen for emotional distress, and is also designed specifically for research with the non-psychiatric population regarding the relationship between depressive symptoms and other variables (Devins \& Orme, 1985). The CES-D assesses the frequency of cognitive, affective and behavioural depressive symptoms based on self-report. The CES-D has 20 items each rated on a 4-point scale with a total score of 0 to 60 . Scores greater than 31 indicate severe depression and scores of 21 to $30.5,16$ to 20.5 and below 15.5 indicate moderate depression, mild depression and no depressive state, respectively (Barnes \& Prosen, 1984). The CES-D has good internal consistency (Cronbach's alpha ranging from 0.84 to 0.90) (Radloff, 1977).

Hospital Anxiety and Depression Scale (HADS). The HADS (Zigmond \& Snaith, 1983) is not as comprehensive as the CES-D but was selected as a second measure of emotional functioning because it specifically evaluates anxiety as well as depression. The HADS is a 14-item self-report questionnaire with each item scored on a 4-point scale. Scoring generates an anxiety subscale and a depression subscale, both with scores ranging from 0 to 21 . Higher scores reflect a greater severity of anxiety or depressive symptoms $(0-7=$ normal; $8-10=$ mild, $11-14=$ moderate , and $15-21=$ severe anxiety or depression). Internal consistency (Cronbach's alpha) of the HADS depression scale in different normative samples varied between 0.81 and 0.90 and the HADS anxiety scale varied between 0.80 and 0.93 (Snaith \& Zigmond, 1994). Test-retest reliability at 2 weeks was found to be acceptable $(r>0.80)$ (Snaith \& Zigmond, 1994). The HADS has good sensitivity and specificity for detecting changes in anxiety and depression (Herrmann, 1997).

Community Integration Questionnaire (CIQ). The CIQ (Willer et al., 1993) is one of the most commonly used measures of community integration following TBI which measures participants' participation and involvement in the community following discharge from hospital. The CIQ is divided into three subscales: home competency (5 items), social integration (5 items) and productive activity (3 items). Participants' scores on the home competency and social integration subscales range from 0 to 10 , and the productive activity subscale ranges from 0 to 15 , with total CIQ scores ranging from 0 to 25 , whereby higher scores indicate higher levels of community integration. The CIQ has high test-retest reliability ( $r=0.91$ for participants' ratings and $r=0.97$ for family members' ratings), good internal consistency and similar ratings between clients and their family members (Kuipers, Kendall, Fleming, \& Tate, 2004; Willer et al., 1993) suggesting that clients provide relatively reliable self-ratings of community integration. There is also evidence supporting the concurrent validity of the CIQ with other outcome measures of disability (Sander, Fuchs, High, Hall, Kreutzer, \& Rosenthal, 1999). In this study, the revised method for scoring the CIQ recommended by Sander et al. was used. CIQ total scores were compared to the average scores reported by Sander et al. based on a sample of 312 people with TBI $(M=14.26, S D=5.52)$.

\section{Procedure}

Ethical clearance for the project was approved by the relevant hospital and university ethics committees prior to beginning the study. Eligible participants were invited to participate in the study by their treating occupational therapist prior to discharge from the inpatient rehabilitation ward. The participants were interviewed in the week prior to discharge from the inpatient brain injury rehabilitation unit and 2 months following discharge. In the first interview, participants completed the SADI, HADS and CES-D. These three measures were repeated at the second interview in addition to the CIQ. The duration of each assessment session was approximately 1 hour. The participants' treating occupational therapist completed the therapists' SADI checklist on discharge and this information was used to assist in assigning SADI scores. The participants were interviewed by one of three Graduate Entry Masters Occupational Therapy students from the University of Queensland or a qualified occupational therapist. The assessments prior to discharge took place in a quiet room at the hospital. Postdischarge interviews were also conducted in a quiet room at the hospital while participants were attending outpatient rehabilitation or on a separate visit to the hospital. In cases where the participants were unable to attend the postdischarge interview at the hospital (e.g., due to distance) the questionnaires were sent to them in the mail with a reply-paid envelope to complete and a telephone interview was conducted to administer the SADI. If necessary, assistance was provided over the telephone to complete the other questionnaires. 


\section{Data Analysis}

Pre- and postdischarge levels of self-awareness according to the SADI and levels of emotional functioning according to the HADS and CES-D were examined initially using frequencies. Total CIQ score was interpreted according to the normative data reported by Sander et al. (1999).

Nonparametric statistics, including the Wilcoxon Signed Ranks Test and Spearman's correlation, were used to analyse the data due to the small sample size, skewed distributions and ordinal measures used to assess self-awareness and emotional distress. Wilcoxon Signed Ranks Tests were used to compare the levels of self-awareness and emotional distress between the two time periods (predischarge and 2 months postdischarge) on the measures (SADI, HADS, and CES-D). To examine the associations between self-awareness and emotional status variables at both assessment times, Spearman's correlations were used. The relationship between three different subscales of community integration (home integration, social integration, and productivity) and CIQ total score with the self-awareness and emotional distress variables from both assessment times were also examined using Spearman's correlations.

\section{Results}

\section{Levels of Self-Awareness, Emotional Functioning and Community Integration}

Level of self-awareness was examined in terms of frequency of participants in the categories generated from the total SADI scores. Prior to discharge, there was 1 participant $(2.9 \%)$ in the intact selfawareness category (score $=0$ ), and 9 participants $(26.5 \%)$ with mild impairment $($ score $=1-3), 15$ participants $(44.1 \%)$ with moderate impairment (score $=4-6)$, and 9 participants $(26.5 \%)$ with severe impairment of self-awareness (score $=7-9$ ). At 2 months postdischarge, the proportions had changed to 4 participants $(12.5 \%)$ with intact selfawareness, 19 (59.4\%) with mild impairment, 4 $(12.5 \%)$ with moderate impairment, and $5(15.6 \%)$ with severe impairment of self-awareness, out of the 32 participants for whom follow-up SADI data were collected.

Emotional functioning was examined using the HADS and CES-D. Table 2 depicts the proportion of participants at each level of emotional functioning on these measures. The majority of participants was in the normal range or displayed mild levels of emotional distress at both assessments. However, the proportion of participants displaying some level of anxiety or depression

\section{TABLE 2}

Level of Emotional Functioning Pre- and Postdischarge on the Hospital Anxiety and Depression Scale (HADS) and the Center for Epidemiological Studies (CES) Depression Scale for Participants with TBI $(N=34)$

\begin{tabular}{lcc} 
& $\begin{array}{c}\text { Pre-discharge } \\
(N=34) \\
n(\%)\end{array}$ & $\begin{array}{c}\text { Post-discharge } \\
(N=30) \\
n(\%)\end{array}$ \\
\hline HADS-Anxiety & & \\
Normal & $22(64.7)$ & $19(63.3)$ \\
Mild & $10(29.4)$ & $5(16.7)$ \\
Moderate & $1(2.9 \%)$ & $5(16.7)$ \\
Severe & $1(2.9 \%)$ & $1(3.3)$ \\
HADS-Depression & & \\
Normal & $30(88.2)$ & $23(76.7)$ \\
Mild & $4(11.8)$ & $5(16.7)$ \\
Moderate & - & $2(6.6)$ \\
Severe & - & - \\
CES-Depression & & $16(53.3)$ \\
Normal & $23(65.7)$ & $5(16.7)$ \\
Mild & $3(8.6)$ & $6(20.0)$ \\
Moderate & $6(17.1)$ & $3(10.0)$ \\
Severe & $2(5.7)$ & \\
\hline
\end{tabular}

was slightly greater following discharge. The CES-D detected slightly higher levels of depression than the HADS.

Level of community integration was assessed by self-report for 30 participants at 2 months postdischarge using the CIQ. Mean total CIQ for the TBI sample was $12.9(S D=4.6$, range 4-20). Half of the participants had total CIQ scores equal to or above the mean for the normal population $(n=15)$ with two participants having scores more than one standard deviation above the mean. The other 15 participants (50\%) had total CIQ scores below the mean for the normal population with 7 participants having scores more than 1 standard deviation below the mean, thus indicating a low level of community integration.

\section{Changes in Self-Awareness and Emotional Distress}

Pre- and postdischarge scores on the self-awareness and emotional distress measures are displayed in Table 3. A significant decrease in total SADI scores $(p<.001)$ was found between the pre- and postdischarge assessments indicating gains in level of self-awareness.

The difference between pre- and postdischarge HADS Anxiety scores was not significant. The difference between pre- and postdischarge HADS 
TABLE 3

Comparison Between Predischarge and Postdischarge Measures of Self-Awareness and Emotional Distress Using Wilcoxon Signed Ranks Test

\begin{tabular}{lcccc} 
Measure & $\begin{array}{c}\text { Pre-discharge } M \\
\text { (SD) range }\end{array}$ & $\begin{array}{c}\text { Post-discharge } M \\
\text { (SD) range }\end{array}$ & $z$ & $p$ \\
\hline SADI & $4.9(2.2) 0-9$ & $3.0(2.6) 0-9$ & -3.61 & $<0.01^{* *}$ \\
HADS-A & $5.6(3.9) 0-15$ & $5.8(4.5) 0-15$ & -0.49 & 0.62 \\
HADS-D & $3.8(2.5) 0-9$ & $4.4(3.3) 0-11$ & -1.69 & 0.09 \\
CES-D & $14.9(9.0) 0-37$ & $16.8(8.6) 3-35$ & -1.73 & 0.08 \\
\hline
\end{tabular}

Note: ${ }^{*} p<.05,{ }^{* *} p<.01$.

SADI =Self-awareness of Deficits Interview total score; HADS-A = Hospital Anxiety and Depression Scale Anxiety score; HADS-D = Hospital Anxiety and Depression Scale Depression score; CES-D = Center for Epidemiological Studies -

Depression score.

depression scores approached significance $(p=.09)$ with slightly higher levels of depressive symptoms reported following discharge although the mean score was in the normal range on both occasions (see Table 3). The difference between pre- and postdischarge depression scores on the CES-D scale also approached significance $(p=.08)$ with the mean score for participants in the upper end of the normal range for depression predischarge and in the mild range of depression postdischarge. However, overall, the mean scores on the measures of emotional functioning increased only slightly from predischarge to postdischarge and the differences were not significant.

\section{Correlation between Self-Awareness and Emotional Distress}

Using Spearman's correlation, there was no significant relationship between total SADI score and the measures of emotional functioning at predischarge or at the 2-month postdischarge follow-up.

\section{Self-Awareness, Emotional Functioning and Community Integration}

The self-awareness and emotional functioning variables measured at predischarge and at 2-months postdischarge were not significantly correlated with the CIQ subscales or CIQ total score 2 months postdischarge.

\section{Discussion}

This study investigated the development of selfawareness and changes in emotional functioning and relationship between these variables and community integration in adults with TBI during the transition from hospital to the community. The first hypothesis that levels of self-awareness and emotional distress would increase over the period of transition from hospital to the community was partially supported. Self-awareness increased over this period on the SADI. There was no significant increase in level of anxiety or depression, although an increase in depression was observed which approached significance on both measures. The second hypothesis that greater self-awareness would be associated with higher levels of emotional distress was not supported. A third hypothesis that increased self-awareness would be associated with higher levels of integration into the community was also not supported.

The first aim of the study was to describe the levels of self-awareness and emotional functioning in adults with TBI during transition from hospital to the community. According to the SADI, nearly all participants showed some impairment of self-awareness prior to discharge with the majority $(44 \%)$ rated as having a moderate impairment of self-awareness. At 2 months follow-up, there was a general shift in SADI scores towards higher levels of self-awareness with the majority of participants (59\%) rated as having a mild impairment of self-awareness. It is important to note that despite this trend towards improved self-awareness there was a spread of participants across all levels of self-awareness at both times, with almost $16 \%$ of the sample displaying a persisting and severe impairment of selfawareness following transition into the community. It is this group, while in the minority, who could be expected to have limited success in outpatient or community based rehabilitation.

The sample predominantly displayed few symptoms of anxiety or depression on the HADS and those that did were mostly in the mild range on both assessments. The CES-D detected a higher rate of depressive symptoms on both occasions but more than half of the participants were 
still in the normal range. The differences in rate of classification of depression between the CES-D and HADS may be due to differences in the overlap between TBI sequelae and the depressive symptoms on the questionnaires. At least 7 of the 20 items on the CES-D overlap with TBI sequelae, whilst 2 of the 7 items on the HADS depression scale ('I can enjoy a good book or radio or TV program' and 'I feel as if I am slowed down') may be symptomatic of TBI as well as depression (Babin, 2003). This highlights some ambiguity in evaluating level of depression in the TBI population.

The second aim of the study was to determine changes in level of self-awareness and emotional functioning during the transition phase. As hypothesised, self-awareness measured by the SADI significantly increased after discharge. This result support previous research findings that selfawareness develops with time (Fischer et al., 2004; Fleming et al., 2002; Godfrey et al., 1993). In relation to changes in level of emotional functioning, although there was a slightly higher level of depression after discharge this did not reach statistical significance.

Third, the study aimed to examine the relationship between self-awareness and emotional functioning. In contrast to previous studies (Fleming \& Strong, 1995; Fordyce, Roueche, \& Prigatano, 1983; Godfrey et al., 1993; Malec, Machulda, \& Moessner, 1997), there were no significant correlations between the SADI and the measures of emotional functioning. As the current study was conducted with participants at a relatively early stage postinjury, the nonsignificant findings may suggest that it takes some time in the community for individuals with greater self-awareness to develop signs of emotional distress. It is possible that at 2 months postdischarge the participants were still quite optimistic about future recovery and this is reflected in the low incidence of depression and anxiety in the sample. Further longitudinal research is needed to determine patterns of self-awareness and emotional distress at later stages following discharge. Research with larger samples may also investigate the causal nature of any observed relationship between self-awareness and emotional distress. Although intuitively one would expect that recognition of more problems would lead to greater emotional distress, this may not be the case (Prigatano, 2005). Potential factors mediating this relationship, such as coping style and social support, need to be investigated.

A final aim of the study was to investigate the relationship between self-awareness and level of community integration. The hypothesis that increased self-awareness would be associated with better community integration was not consistently supported. This finding is in contrast to previous research that has found associations between self-awareness and aspects of community integration (Ben-Yishay et al., 1987; Malec \& Moessner, 2000; Ownsworth \& McFarland, 2004; Sherer et al., 1998a; Trudel et al., 1998). This may reflect the fact that outcome, particularly in the early phase following TBI, is multifaceted and is determined by a variety of neurological, psychological and environmental factors, of which level of self-awareness may be just one contributing factor (Fleming et al., 1998). Another possible explanation may be the self-report nature of the CIQ, whereby people who under-report problems may also over-report level of involvement in home, social or productive activities. Although previous research has not found significant differences between proxy-ratings and self-ratings on the CIQ (Kuipers et al., 2004; Willer et al., 1993), the use of proxy-ratings in future research may help overcome the possibility of participants overreporting level of community integration.

\section{Implications for Treatment}

The significant increase in self-awareness at 2 months following discharge supports the view that accuracy of self-appraisal increases over time when the client is faced with real life experiences, as suggested by Fleming et al. (2002). It is believed that a degree of self-awareness is necessary for a person to be motivated in rehabilitation and to set achievable rehabilitation goals. Fischer et al. (2004) identified that low motivation, resulting from deficits in self-awareness, was an influential factor for treatment compliance and rehabilitation outcomes. During inpatient rehabilitation, clients are exposed to a somewhat artificial environment that may not provide sufficient opportunity to encounter real-life situations and to explore limitations. Once the client is living in the community and does not have the support of staff and the structured hospital environment, he or she may begin to encounter unexpected difficulties with daily life tasks and begin to recognise the extent of disability (Dirette, 2002).

The lack of significant correlations between level of self-awareness and anxiety and depression in this study suggests that the process of acknowledging problems is not necessarily associated with increased emotional distress, at least in the shortterm. It is important to note that the majority of participants experienced symptoms of anxiety and depression in the normal range, and where clinically significant anxiety or depression was detected, it was mostly in the mild range. This 
suggests that although some patients may experience increased emotional distress, this is not usually at a debilitating level, and should not present a major deterrent to working with clients to enhance level of self-awareness. However, there was a small group of participants who reported symptoms of anxiety and depression in the severe range, and this group may be at greater risk of poor adjustment as self-awareness develops. Therefore, postdischarge follow-up is important to monitor individuals' emotional status with intervention provided during outpatient rehabilitation to assist development of self-awareness using approaches that protect individuals from heightened emotional distress (for a review of awareness interventions see Fleming \& Ownsworth, in press). This supportive process may improve motivation and compliance with rehabilitation, enhance clients' functional outcomes and lead to more successful community integration (Fleming et al., 2002; Malec \& Basford, 1996).

Previous research (Ben-Yishay et al., 1987; Malec, Brown, \& Moessner, 2004) has found that holistic neuropsychological day treatment programs that were designed to improve clients' awareness and acceptance of their deficits enhanced employment outcomes. Components of these programs involved individual and group cognitive rehabilitation, small group procedures addressing psychosocial issues, and the involvement of significant others. All participants in the current study received some form of postdischarge support such as regular outpatient therapy appointments to community-based services. However this varied in type, length and intensity between participants, and may have been a confounding factor in the development of self-awareness and emotional functioning for the participants in this study. Future research is required to investigate the impact of various types of postdischarge support on self-awareness, emotional status and community integration.

Participants with TBI in this study displayed varied levels of impaired self-awareness ranging from no impairment through to severe impairment. It is suggested that different intervention strategies be implemented for clients according to their levels of self-awareness. For example, clients with very low levels of self-awareness may benefit from engagement in rehabilitation activities that facilitate development of self-awareness of deficits, or 'reality-testing' activities (Fleming et al., 1998). However, clients with partial selfawareness, who may also be employing defensive coping strategies such as denial, may benefit more from psychotherapy and supportive counselling
(Katz et al., 2002; Ownsworth, 2005). Other interventions for this group of clients may include behavioural therapy, the use of psychoeducation regarding deficits, goal setting, and positive feedback on improvement in performance (Barco, Crossen, Bolesta, Werts, \& Stout, 1991; Fleming et al., 1998; Lucas \& Fleming, 2005; Sherer et al., 1998b). However, the use of confrontational approaches to allow clients to experience their limitations may cause distress in clients with defensive denial and be counterproductive (Moore, Stambrook, \& Peters, 1989).

It is recommended that rehabilitation professionals be sensitive to clients' responses during therapy activities designed to enhance self-awareness and monitor their readiness for different approaches. Likewise, it is important to be mindful that after discharge clients may be exposed to the extent of their disability on a daily basis and require support to assist with adjustment and coping in this phase. LoBello et al. (2003) highlighted the importance of establishing a therapeutic alliance with clients in rehabilitation to develop skills necessary for social relationships and forming new relationships within the community. If rehabilitation does not focus on the development of these skills, an individual may become '... isolated, become dissatisfied with life and develop a myriad of additional psychological problems' (LoBello et al., 2003 , p. 294). Therefore, it is optimal for intervention to start early in rehabilitation and continue on a long-term support basis after discharge.

\section{Limitations of the Study}

The current study is limited by the small sample size, which may have affected the validity of the results. Further studies should attempt to include a larger number of participants. Recruiting participants from several different centres would increase the sample size and generalisability of the results.

In this study, we used only one measure of self-awareness, although the use of multiple measures has been recommended in the past (Simmond \& Fleming, 2003a). Other methods of evaluating self-awareness include the comparison of patient self-ratings of their performance with ratings by significant others, for example using the Patient Competency Rating Scale (Prigatano, Fordyce, Zeiner, Roueche, Pepping, \& Wood, 1986) or the Head Injury Behaviour Scale (Godfrey et al., 1993). Use of comparison methods was considered problematic in the current study given that it would be questionable to use ratings by family members prior to hospital discharge, when family has had little experience 
living with the person with TBI on a daily basis. It was possible to get therapists' ratings of the participants' performance before discharge, but not all participants were returning to the hospital for outpatient rehabilitation, and it was considered methodologically inadvisable to compare therapist's ratings before discharge with family's ratings at follow-up.

As mentioned earlier, the 2-month follow-up period was relatively short and further increases in anxiety and depression may result with time, as clients are exposed on a more prolonged basis to their limitations at home and in the community. Participants were first seen before discharge from inpatient rehabilitation at an average of approximately 2 months postinjury with all participants less than 6 months post-injury. Previous studies found a significant increase in emotional distress after periods of 6 months, 1 year and 2 to 3 years post-injury (Fleming et al., 1998; Fordyce et al., 1983; Godfrey et al., 1993). Lezak (1987) and Lezak and O'Brien (1988) found that anxiety and depression were highest between 7 months and 12 months post-injury. Thus, further research using later follow-ups may be fruitful for examining the relationship between self-awareness, emotional functioning and community integration.

\section{Conclusions}

This study found that self-awareness increased over the first 2 months after discharge from brain injury rehabilitation. Level of self-awareness was not associated with levels of anxiety and depression, which were mostly not in the clinical range. The need for support during the early period of community integration when self-awareness is increasing as the individual is faced with real-life difficulties was identified. Different types of support were recommended for clients with varying levels of self-awareness. Further research is needed to review current treatment approaches and compare their effectiveness in increasing level of self-awareness and minimising any associated emotional distress. The effectiveness of continuing community support after discharge from hospital for assisting adjustment, increasing functional outcomes and facilitating community reintegration should be evaluated.

\section{Acknowledgements}

The authors gratefully acknowledge the assistance of patients and staff of the Occupational Therapy Department at Princess Alexandra Hospital. This research was supported by grants from the School of Health and Rehabilitation Sciences Motor Accident Insurance Commission Grant scheme and the Princess Alexandra Hospital Research and Development Foundation.

\section{References}

Andersson, S., Gundersen, P.M., \& Finset, A. (1999). Emotional activation during therapeutic interaction in traumatic brain injury: Effect of apathy, selfawareness and implications for rehabilitation. Brain Injury, 13(6), 393-404.

Babin, P.R. (2003). Diagnosing depression in persons with brain injuries: A look at theories, the DSM-IV and depression measures. Brain Injury, 17, 889-900.

Barco, P.P., Crossen, B., Bolesta, M.M., Werts, D., \& Stout, P. (1991). Training awareness and compensation in post-acute hand injury rehabilitation. In J. S. Kreutzer \& P.H. Wehman (Eds.), Cognitive Rehabilitation for persons with traumatic brain injury: A functional approach (pp. 129-146). Baltimore, MD: Paul H. Brookes.

Barnes, G.E. \& Prosen, H. (1984). Depression in Canadian general practice attenders. Canadian Journal of Psychiatry, 29, 2-10.

Ben-Yishay, Y., Silver, S. M., Piasetsky, E., \& Rattok, J. (1987). Relationship between employability and vocational outcome after intensive holistic cognitive rehabilitation. Journal of Head Trauma Rehabilitation, 2(1), 35-48.

Brown, M., Gordon, W. A., \& Spielman, L. (2003). Participation in social and recreational activity in the community by individuals with traumatic brain injury. Rehabilitation Psychology, 48(4), 266-274.

Devins, G.M., \& Orme, C.M. (1985). Center for epidemiologic studies depression scale. In D.J. Keyer \& R.C. Sweetlands (Eds.), Test Critiques (Vol. 2, pp. 144-160). Kansas City, KS: Westport Publication.

Dirette, D. (2002). The development of awareness and the use of compensatory strategies for cognitive deficits. Brain Injury, 16(10), 861-871.

Fischer, S., Gauggel, S., \& Trexler, L.E. (2004). Awareness of activity limitations, goal setting and rehabilitation outcome in patients with brain injuries. Brain Injury, 18(6), 547-562.

Fleming, J.M., Connell, J., Tooth, L., \& Strong, J. (2002). A comparison of adjustment and self-awareness in adults after traumatic brain injury and spinal cord injury: The transition from hospital to home. The Journal of Cognitive Rehabilitation, 20(3), 28-36.

Fleming, J., \& Ownsworth, T. (In press). A review of awareness interventions in brain injury rehabilitation. Neuropsychological Rehabilitation.

Fleming, J.M., \& Strong, J. (1995). Self-awareness of deficits following acquired brain injury: Considerations for rehabilitation. British Journal of Occupational Therapy, 58(2), 55-60. 
Fleming, J.M., \& Strong, J. (1997). The development of insight following severe traumatic brain injury: Three case studies. British Journal of Occupational Therapy, 60(7), 295-300.

Fleming, J.M., \& Strong, J. (1999). A longitudinal study of self-awareness: Functional deficits underestimated by persons with brain injury. The Occupational Therapy Journal of Research, 19(1), 3-17.

Fleming, J.M., Strong, J., \& Ashton, R. (1996). Selfawareness of deficits in adults with traumatic brain injury: How best to measure? Brain Injury, 10(1), $1-15$.

Fleming, J.M., Strong, J., \& Ashton, R. (1998). Cluster analysis of self-awareness levels in adults with traumatic brain injury and relationship to outcome. The Journal of Head Trauma Rehabilitation, 13(5), 39-52.

Fordyce, D.J., \& Roueche, J.R. (1986). Changes in perspectives of disability among patients, staff, and relatives during rehabilitation of brain injury. Rehabilitation Psychology, 31, 217-229.

Fordyce, D.J., Roueche, J.R., \& Prigatano, G.P. (1983). Enhanced emotional reactions in chronic head trauma patients. Journal of Neurology, Neurosurgery, and Psychiatry, 46, 620-624.

Giacino, J.T., \& Cicerone, K.D. (1998). Varieties of deficit unawareness after brain injury. The Journal of Head Trauma Rehabilitation, 13(5), 1-15.

Godfrey, H.P.D., Partridge, F.M., Knight, R.G., \& Bishara, S. (1993). Course of insight disorder and emotional dysfunction following closed head injury: A controlled cross-sectional follow-up study. Journal of Clinical and Experimental Neuropsychology, 15(4), 503-515.

Herrmann, C. (1997). International experiences with the hospital anxiety and depression scale: A review of validation data and clinical results. Journal of Psychosomatic Research, 42(1), 17-41.

Katz, N., Fleming, J., Hartman-Maeir, A., Keren, N., \& Lightbody, S. (2002). Unawareness and/or denial of disability: Implications for occupational therapy intervention. Canadian Journal of Occupational Therapy, 69(5), 281-291.

Kuipers, P., Kendall, M., Fleming, J., \& Tate, R. (2004). Comparison of the Sydney Psychosocial Reintegration Scale (SPRS) with the Community Integration Questionnaire (CIQ): Psychometric properties. Brain Injury, 18, 161-177.

Langer, K.G., \& Padrone, F.J. (1992). Psychotherapeutic treatment of awareness in acute rehabilitaion of traumatic brain injury. Neuropsychological Rehabilitation, 2(1), 59-70.

Lanham, R.A., Weissenburger, J.E., Schwab, K.A., \& Rosner, M.M. (2000). A longitudinal investigation of the concordance between individuals with traumatic brain injury and family or friend ratings on the Katz Adjustment Scale. The Journal of Head Trauma Rehabilitation, 15(5), 1123-1139.

Lezak, M.D. (1987). Relationships between personality disorders, social disturbances, and physical disability following traumatic brain injury. Journal of Head Trauma Rehabilitation, 2(1), 57-69.

Lezak, M.D., \& O’Brien, K.P. (1988). Longitudinal study of emotional, social, and physical changes after traumatic brain injury. Journal of Learning Disability, 21(8), 456-463.

LoBello, S.G., Underhil, A.T., Valentine, P.V., \& Stroud, T.P. (2003). Social integration and life and family satisfaction in survivors of injury at 5 years postinjury. Journal of Rehabilitation and Development, 40(4), 293-298.

Lucas, S., \& Fleming, J.M. (2005). Interventions for improving self-awareness following acquired brain injury: A review of the literature. Australian Occupational Therapy Journal, 52, 160-170.

Malec, J.F., \& Basford, J.S. (1996). Postacute brain injury rehabilitation. Archives of Physical Medicine and Rehabilitation, 77, 198-207.

Malec, J.F., Brown, A.W., \& Moessner, A.M. (2004). Personality factors and injury severity in the prediction of early and late traumatic brain injury outcomes. Rehabilitation Psychology, 49(1), 55-61.

Malec, J.F., Machulda, M.M., \& Moessner, A.M. (1997). Differing problem perceptions of staff, survivors, and significant others after brain injury. Journal of Head Trauma Rehabilitation, 12(3), $1-13$.

Malec, J.F., \& Moessner, A.M. (2000). Self-awareness, distress, and postacute rehabilitation outcome. Rehabilitation Psychology, 45(3), 227-241.

Malia, K. (1997). Insight after brain injury: What does it mean? The Journal of Cognitive Rehabilitation, 15(3), 10-16.

McGlynn, S.M., \& Schacter, D.L. (1989). Unawareness of deficits in neuropsychological syndromes. Journal of Clinical and Experimental Neuropsychology, 11, 143-205.

Moore, A.D., Stambrook, M., \& Peters, L.C. (1989). Coping strategies and adjustment after closed head injury: A cluster analytical approach. Brain Injury, 3, 171-175.

Ownsworth, T.L. (2005). The impact of defensive denial upon adjustment following traumatic brain injury. Neuro-psychoanalysis, 7, 83-94.

Ownsworth, T.L., \& McFarland, K. (2004). Investigation of psychological and neuropsychological factors associated with clinical outcome following a group rehabilitation programme. Neuropsychological Rehabilitation, 14, 535-562.

Prigatano, G.P. (1992). Personality disturbances associated with traumatic brain injury. Journal of Consulting and Clinical Psychology, 60(3), 360-368.

Prigatano, G.P. (2005). Disturbances of self-awareness and rehabilitation of patients with traumatic brain injury: A 20-year perspective. Journal of Head Trauma Rehabilitation, 20, 19-29.

Prigatano, G.P., Fordyce, D.J., Zeiner, H.K., Roueche, J.R., Pepping, M., \& Wood, B.C. (1986). Neuropsychological rehabilitation after brain 
injury. Baltimore, MD: John Hopkins University Press.

Prigatano, G.P., \& Schacter, D.L. (1991). Introduction. In G.P. Prigatano \& D.L. Schacter (Eds.), Awareness of deficit after brain injury: Clinical and theoretical issues. New York: Oxford University Press.

Radloff, L.S. (1977). The CES-D scale: A new selfreport depression scale for research in the general population. Applied Psychological Measurement, 1, 385-401.

Sander, A.M., Fuchs, K.L., High, W.M., Hall, K.M., Kreutzer, J.S., \& Rosenthal, M. (1999). The community integration questionnaire revisited: An assessment of factor structure and validity. Archives of Physical Medicine and Rehabilitation, 80, 1303-1308.

Sherer, M., Bergloff, P., Levin, E., High, W.M., Oden, K.E., \& Nick, T.G. (1998a). Impaired awareness and employment outcome after traumatic brain injury. Journal of Head Trauma Rehabilitation, 13(5), 52-61.

Sherer, M., Hart, T., Nick, T.G., Whyte, J., Thompson, R.N., \& Yablon, S.A. (2003). Early impaired selfawareness after traumatic brain injury. Archives of Physical Medicine Rehabilitation, 84, 168-176.

Sherer, M., Oden, K., Bergloff, P., Levin, E., \& High, W.M. (1998b). Assessment and treatment of impaired awareness after brain injury: Implications for community re-integration. Neurorehabilitation, $10,25-37$.
Simmond, M. \& Fleming, J. (2003a). Occupational therapy assessment of self-awareness following traumatic brain injury: A literature review. British Journal of Occupational Therapy, 66(10), 447-453.

Simmond, M. \& Fleming, J. (2003b). Reliability of the self-awareness of deficits interview for adults with traumatic brain injury. Brain Injury, 17(4), 325-337.

Snaith, R.P., \& Zigmond, A.S. (1994). The Hospital Anxiety and Depression Scale manual. Windsor, Berkshire, UK: NFER-NELSON.

Tate, R., Hodgkinson, A., Veerabangsa, A., \& Maggiotto, S. (1999). Measuring psychosocial recovery after traumatic brain injury: Psychometric properties of a new scale. Journal of Head Trauma Rehabilitation, 14, 543-557.

Trudel, T.M., Tryon, W.W., \& Purdum, C.M. (1998). Awareness of disability and long-term outcome after traumatic brain injury. Rehabilitation Psychology, 43, 267-281.

Wallace, C.A., \& Bogner, J. (2000). Awareness of deficits: Emotional implications for persons with brain injury and their significant others. Brain Injury, 14(6), 549-562.

Willer, B., Rosenthal, M., Kreutzer, J.S., Gordon, W.A., \& Rempel, R. (1993). Assessment of community integration following rehabilitation for traumatic brain injury. Journal of Head Trauma Rehabilitation, 8(2), 75-87.

Zigmond, A.S., \& Snaith, R.P. (1983). The hospital anxiety and depression scale. Acta Psychiatrica Scandinavica, 67, 361-370. 